\title{
OBSERVATIONS OF HYDROCARBON EMISSION IN DISKS AROUND YOUNG STARS
}

\author{
B. Acke ${ }^{1,2}$
}

\begin{abstract}
I give an overview of the recent scientific results based on observations of PAH emission from circumstellar disks around young stars. The stellar radiation field plays a key role in the excitation and destruction of the PAH molecules in the disk. The detection rate of PAH emission in disks is optimal for stars of spectral type A. Around stars of similar temperature, the disk structure determines the PAH emission strength: disks with a flared geometry produce stronger $\mathrm{PAH}$ emission than flattened disks. The spectral properties of the emission features, indicative of the chemistry of the emitting hydrocarbons, is closely linked to the central star radiation field. The main PAH features shift to redder wavelengths with decreasing stellar effective temperature. This trend has been interpreted as an indication for a higher aliphatic/aromatic ratio of the hydrocarbon mixture around cool stars with respect to hot stars. An alternative explanation may be a more significant contribution to the infrared emission of very small grains around cooler stars.
\end{abstract}

\section{Introduction}

As a result of the star-formation process, young stars are surrounded by circumstellar disks. These disks, rich in gas and dust, are believed to be the birthplaces of planetary systems. The disk dissipation timescale is short; In only a few million years, half of the newly formed stars become diskless (e.g., Fedele et al. 2010). It is in this short time span that processes such as dust grain growth, crystallisation, sedimentation, photoevaporation, and chemical evolution can take place before the dust is removed. They affect the appearance of the system throughout the electromagnetic spectrum.

\footnotetext{
1 Instituut voor Sterrenkunde, University of Leuven, Celestijnenlaan 200D, 3001 Leuven, Belgium; e-mail: bram@ster.kuleuven.be

2 Postdoctoral Fellow of the Fund for Scientific Research, Flanders
} 
Young stars are traditionally categorized according to their spectral type. $\mathrm{T}$ Tauri stars are young analogues of the Sun, with spectral type later than mid-F and main-sequence masses $\mathrm{M}_{\star} \leq 1.5 M_{\odot}$. Higher-mass young stars are called Herbig Ae/Be stars (Herbig 1960). Observations indicate that T Tauri and Herbig Ae stars follow a similar disk evolution. Evidence is growing, however, that disks around Herbig Be stars $\left(\mathrm{M} \geq 3 M_{\odot}\right.$, spectral type earlier than mid-B) are qualitively different in structure. In the following, it will become clear that the T-Tauri, Herbig-Ae and Herbig-Be classification is also meaningful in the framework of the hydrocarbon emission.

In this Review, I group the scientific results on PAHs in young circumstellar disks in three sections. Section 2 focuses on the detection rates of disk PAH emission features for different types of young stars. In Section 3, it is shown that the strength of the PAH emission is linked to the disk geometry. Finally, I summarize the chemical properties of the hydrocarbons, in relation to the stellar radiation field, in Section 4.

\section{PAH detection rates in disks}

The detection rate of IR emission features produced by $\mathrm{PAH}$ molecules in the circumstellar disk is low for T Tauri stars $(\sim 10 \%)$ and Herbig Be stars $(<35 \%)$. More than two thirds of the intermediate-mass Herbig Ae stars, on the other hand, have detected PAH emission from disks.

\subsection{T Tauri stars}

Geers et al. (2006) and Geers (2007) searched for PAH emission in the spectra of $37 \mathrm{~T}$ Tauri stars obtained with the Infrared Spectrograph aboard the Spitzer Space Telescope (Spitzer-IRS; Houck et al. 2004). They determined a detection rate of 11-14\%, with no detections for stars with spectral type later than G8. Furlan et al. (2006) studied Spitzer-IRS spectra of young T Tauri disks in the Taurus star-forming region, whereas Oliveira et al. (2010) investigated young stellar objects in the Serpens Molecular Cloud. Only 3-4\% of the disk sources have detected PAH features. One could think that the weak UV field of a T Tauri star is insufficient to excite the PAH molecules in the disk. However, Geers et al. (2006) compare their data to a simple model for PAH emission in a circumstellar disk. They assume PAH molecules containing 100 carbon atoms $\left(\mathrm{N}_{\mathrm{C}}=100\right)$. To explain the low detection rate, the authors conclude that the abundance of gasphase PAHs in disks around T Tauri stars must be 10-100 times lower than the PAH abundance of the general interstellar medium (ISM; Cesarsky et al. 2000; Habart et al. 2001).

Geers et al. (2009) searched for PAH emission in low-mass embedded protostars, the evolutionary stage preceding the $\mathrm{T}$ Tauri stage, and determined an even lower detection rate $(2 \%)$. The authors argue that absorption by the envelope surrounding the star and disk is not sufficient to explain the absence of features, 
and again suggest that lower PAH abundances are needed. They hypothesize that PAHs enter the disk either frozen out on dust grains, or in agglomerates. Quanz et al. (2007) studied the Spitzer-IRS spectra of a sample containing 14 FU Orionis stars. The latter are young low-mass objects that undergo energetic accretion events, leading to strong outbursts at optical and near-IR wavelengths. Only one object, possibly even misclassified as a FU Orionis star, shows hydrocarbon emission features, amongst others the feature at $8.2 \mu \mathrm{m}$. The low detection rate in this sample $(\leq 7 \%)$ supports the hypothesis of $\mathrm{PAH}$ underabundance in the disks around young low-mass stars.

Siebenmorgen \& Krügel (2010) modeled the effect of hard radiation on PAH molecules in a circumstellar disk (see also Siebenmorgen et al., elsewhere in this volume). The authors find that for typical T Tauri X-ray luminosities $\left(L_{X} / L_{\star} \sim\right.$ $10^{-4}-10^{-3}$; Preibisch et al. 2005$)$, PAH molecules in the disk surface are efficiently destroyed at all radial distances, leading to a suppression of the PAH emission. Siebenmorgen \& Krügel (2010) suggest that in the few T Tauri disks with detected PAH emission, turbulent vertical mixing brings PAHs to the upper disk layers faster than photodestruction removes the molecules. The hard radiation component in Herbig Ae stars is much weaker $\left(L_{X} / L_{\star}<10^{-7}\right.$ with a few exceptions; Stelzer et al. 2006). A lower destruction rate is expected, consistent with the much higher detection rates (see Sect. 2.3).

However, it may be that the low detection rate is biased because of the focus on the 6.2 and $11.3 \mu \mathrm{m}$ features. Bouwman et al. (2008) find that 5 out of the 7 lowmass young stars in their sample display hydrocarbon emission features. The features are weak compared to the underlying continuum and silicate emission, and a dedicated fit is required to reveal them. Bouwman et al. show that the hydrocarbon emission spectrum is different from the spectrum seen in higher-mass young stars. It is dominated by a feature at $8.2 \mu \mathrm{m}$, and has weak 6.2 and $11.3 \mu \mathrm{m}$ features. This deviating hydrocarbon spectrum in T Tauri stars is consistent with the picture outlined in Section 4.

\subsection{Herbig Be stars}

The infrared spectra of roughly half of the Herbig Be stars contain PAH emission features (Acke and van den Ancker, unpublished). Due to the strong UV field of the central star, however, most of the PAH emission comes from surrounding clouds, remnants of the natal cloud, rather than from the disk. Verhoeff et al. (A\&A submitted) have studied the extended PAH emission in a sample of 30 Herbig Be stars. None of the emission can unambiguously be attributed to the disk, but in $35 \%$ of the targets, PAH emission from the disk cannot be excluded either. Verhoeff et al. argue that the disks around Herbig Be stars are fundamentally different from those around lower-mass stars in terms of disk geometry: they are radially less extended and vertically flatter. The authors state that, even if PAH molecules are present in the disk surface at ISM abundances, their emission would be insignificant due to the disk geometry, and swamped by the PAH emission from 
the extended cloud. Moreover, the hard radiation field of early-type young stars may shift the PAH destruction radius beyond the disk outer radius, suppressing the PAHs in the disk altogether.

\subsection{Herbig Ae stars}

The intermediate-mass Herbig Ae stars have by far the highest detection rate of $\mathrm{PAH}$ emission in disks. Their stellar radiation field is optimal to excite but not destroy the molecules. Acke \& van den Ancker (2004) studied the infrared spectra of 46 Herbig Ae/Be stars, mainly based on data obtained with the Short-Wavelength Spectrometer aboard the Infrared Space Observatory (ISO-SWS; de Graauw et al. 1996; Kessler et al. 1996). The detection rate of PAH emission features is $57 \%$. Recently, Acke et al. (2010) investigated a sample of 53 Herbig Ae stars, observed with Spitzer-IRS. The authors detect PAH features in $70 \%$ of the targets. The ISO-SWS and Spitzer-IRS sample overlap in 27 sources. A closer look at this subsample shows that the higher detection rate in the Spitzer-IRS spectra is simply due to the superior sensitivity of the latter instrument.

Because of the high detection rate in Herbig Ae stars, most studies of PAHs in young disks focus on these targets.

\section{Emission strength and disk geometry}

Spatially resolved observations of PAH emission around young stars have clearly demonstrated the disk origin of the emission. Two techniques are commonly used: long-slit spectroscopy (e.g., van Boekel et al. 2004; Habart et al. 2004b, 2006; Geers et al. 2007) and narrow-band imaging (e.g., Lagage et al. 2006; Doucet et al. 2007). The focus lies on the PAH features that are accessible from the ground, i.e. the 3.3 (L-band), 7.7, 8.6 and $11.3 \mu \mathrm{m}$ (N-band) features. The PAH emission region has a typical extent of tens of AU for the $3.3 \mu \mathrm{m}$ feature to hundreds of AU for the features at longer wavelengths. Habart et al. (2004a) and Visser et al. (2007) have modeled PAH emission from a circumstellar disk. These authors conclude that large (i.e., $\mathrm{N}_{\mathrm{C}}=100$ as opposed to $\mathrm{N}_{\mathrm{C}}=30$ ) PAHs are needed to explain the observed spatial extent.

Lagage et al. (2006) \& Doucet et al. (2007) have imaged the HD 97048 disk in narrow-band filters containing the 8.6 and $11.3 \mu \mathrm{m} \mathrm{PAH}$ features, and at continuum wavelengths in between, using VLT/VISIR (Lagage et al. 2004). They confirm the findings mentioned above. Moreover, the images clearly show the flared geometry of the disk surface: the disk opening angle increases with radial distance to the star, which gives it a bowl-shaped appearance (see Fig. 1). The measured increase of surface height above the midplane with radius follows $H \propto R^{1.26 \pm 0.05}$, in perfect agreement with the predicted value for models in hydrostatic radiative equilibrium (Chiang \& Goldreich 1997; Dullemond et al. 2001). This provides strong observational support for the physical assumptions underlying the flared-disk models.

The disk geometry plays an important role in the apparent strength of the PAH emission features. Comparison between observations and models shows that the 


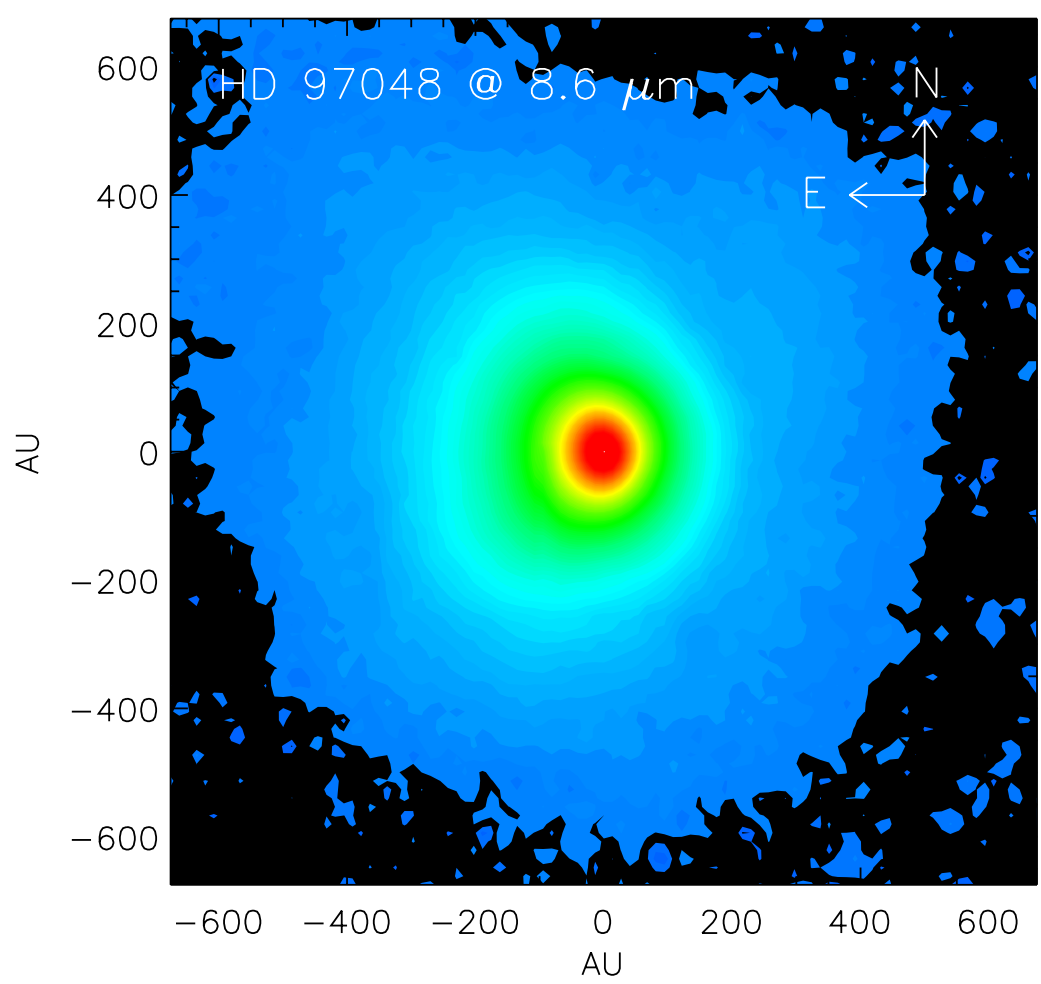

Fig. 1. Image of HD 97048 in a narrow-band filter around the $8.6 \mu \mathrm{m}$ PAH feature (Lagage et al. 2006). The scale is the square-root of the flux to enhance the low-flux regions. Courtesy of E. Pantin and P.-O. Lagage.

far-IR excess of Herbig Ae stars is connected to the degree of flaring of the dust disk. The excess is produced by thermal emission of dust grains in the disk. Strong flaring leads to a large far-IR excess (Meeus et al. 2001; Dullemond \& Dominik 2004; Meijer et al. 2008; Acke et al. 2009). Dullemond et al. (2007) have modeled the effect of dust sedimentation on the appearance of the disk and the strength of the PAH features. Sedimentation makes the dust disk more flattened, and reduces the far-IR excess. However, the gas disk, containing the PAH molecules, remains flared. If dust sedimentation is the dominant process, the models predict stronger PAH emission in flattened disks, both because of an absolute increase in PAH luminosity and an increased feature-to-continuum contrast. Remarkably, the opposite trend is observed: several authors have shown that sources with a flared dust disk have stronger PAH features than those with flattened disks (Meeus et al. 2001; Acke \& van den Ancker 2004; Habart et al. 2004a; Keller et al. 2008; Acke et al. 2010). In Figure 2, the increase of the feature-to-stellar luminosity ratio of the $6.2 \mu \mathrm{m}$ feature with increasing far-IR excess is shown for a sample of Herbig Ae 


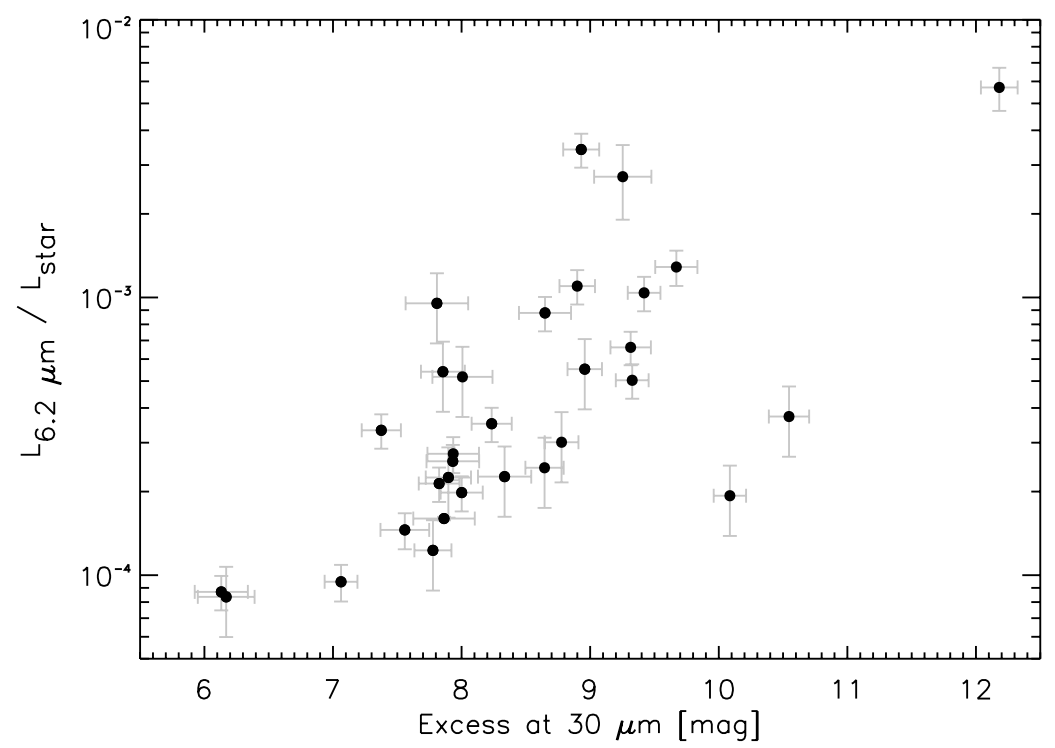

Fig. 2. The feature-to-stellar luminosity ratio of the $6.2 \mu \mathrm{m}$ feature increases with increasing $30 \mu \mathrm{m}$ excess above the stellar photosphere (Acke et al. 2010). Flattened dust disks are characterized by a low, and flared disks by a high far-IR excess.

stars. This correlation has been interpreted as a simple geometric effect: a flared disk has a larger illuminated surface than a flattened disk. However, the link between disk geometry and PAH emission strength is not necessarily a causal link. PAH molecules in the disk surface layer are an important contributor to the gas thermal balance due the photo-electric effect. Meeus et al. (2010) have shown that inclusion of PAH molecules in different charge states can alter the gas temperature considerably. The disk becomes warmer and the degree of flaring larger. PAHs hence help to sculpture the disk structure (see Kamp, elsewhere in this volume).

A few objects with flattened dust disks (i.e. low far-IR excesses) are still strong PAH sources (van der Plas et al. 2008; Fedele et al. 2008; Verhoeff et al. 2010). In those targets, the scenario of dust sedimentation could be at work. The general trend, however, shows that sedimentation is not the dominating process in most disks. It indicates that either dust and gas remain well coupled (e.g. due to strong turbulent motions), or that the PAH molecules coagulate with dust grains and settle along with the dust (Dullemond et al. 2007).

Because of the low detection rate of PAH emission in the lower-mass stars, the statistics in this group of objects is too poor to perform a similar study on disk geometry. Bouwman et al. (2008) note that the T Tauri stars without detected hydrocarbon emission in their sample have a SED consistent with a flattened disk structure. Geers et al. (2006) mention that gaps in the inner dust disk need to be taken into account as well. Such gaps may be formed due to the 


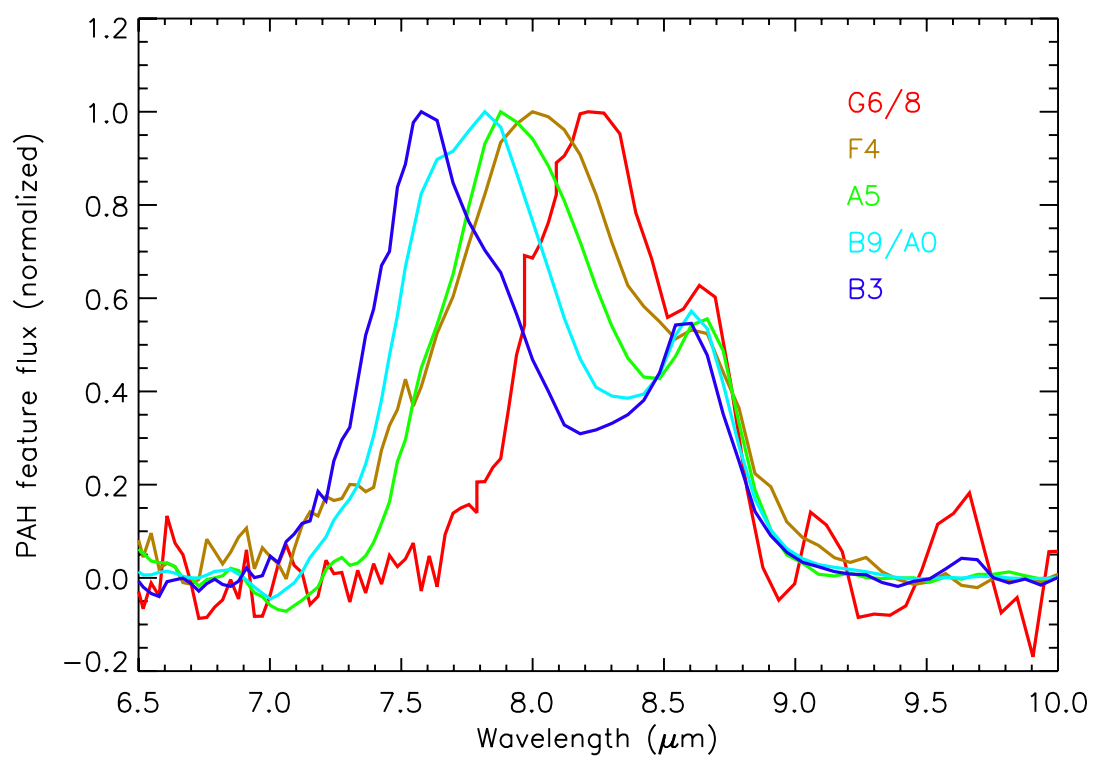

Fig. 3. The $7.7 \mu \mathrm{m}$ feature shifts to longer wavelengths with decreasing stellar effective temperature.

gravitational interaction with a planetary system or a companion star. A gap with an extent of several to tens of AU reduces the thermal continuum emission of the dust at near- and mid-IR wavelengths. This enhances the feature-to-continuum contrast. Moreover, the disk beyond the gap outer radius receives more direct stellar photons. As a result, the disk becomes warmer, giving rise to an increase in scale height, and the surface area exposed to stellar radiation becomes larger than in a gap-less disk. Next to the increased feature-to-continuum contrast, this enhances the absolute PAH luminosity.

\section{Chemistry and stellar radiation field}

Peeters et al. (2002, and elsewhere in this volume) defined three classes of objects based on the peak positions and the relative contribution of the sub-bands of the $\mathrm{PAH}$ features in the 6-9 $\mu \mathrm{m}$ range. Class A contains sources with blue 6.2 and $7.7 \mu \mathrm{m}$ features. These are mostly general ISM sources, HiI regions, and reflection nebulae, including those around Herbig Be stars. Class C sources are characterized by red features. Very little objects belong to this Class: cool carbon-rich evolved stars, red giant stars and a few $\mathrm{T}$ Tauri stars. The Herbig Ae stars belong to Class B (intermediate feature positions), together with most planetary nebulae and a number of binary post-AGB stars. Sloan et al. $(2005,2008)$ note that the 
PAH spectra of Herbig Ae stars are different from those of evolved stars belonging to Class B. Herbig Ae stars display a $7-9 \mu \mathrm{m}$ feature with an extended red wing beyond $8.0 \mu \mathrm{m}$. The authors therefore propose to create a new Class (dubbed $\mathrm{B}^{\prime}$ ) for the latter.

Boersma et al. (2008) show that the spatially resolved spectra of two Herbig Ae stars exhibit Class A (ISM-like) characteristics far from the central star, and Class B characteristics closer in. The authors argue that all the emission emanates from the surrounding nebula, and not from the disk. This shows that chemical changes can occur even in the surrounding cloud.

In young stars, the Peeters classification is linked to the radiation field of the central star: the wavelength position of the 6.2 and $7.7 \mu \mathrm{m}$ features moves to the red with decreasing stellar effective temperature (Sloan et al. 2007; Keller et al. 2008; Acke et al. 2010). In Figure 3, the Spitzer-IRS 6.5-10 $\mu \mathrm{m}$ spectra of five young stars are shown. For clarity, the underlying continuum has been subtracted and the spectra are normalized to the peak flux of the $7.7 \mu \mathrm{m}$ feature. The figure illustrates the gradual shift of the feature position to shorter wavelengths with increasing stellar effective temperature, from Class $\mathrm{C}$ for the $\mathrm{T}$ Tauri star to Class A for the Herbig Be star.

The interpretation of this shift is still a matter of debate. Sloan et al. (2005) suggested that the redshift of the main features indicates a higher aliphatic/ aromatic $\left(\mathrm{sp}^{3} / \mathrm{sp}^{2}\right)$ hybridization of the hydrocarbon molecules. This hypothesis is supported by the laboratory measurements of Pino et al. (2008). Acke et al. (2010) show that in Herbig Ae stars the redshift of the features, or equivalently $\mathrm{T}_{\text {eff }}$, is connected to an increase in relative strength of the features at 6.8 and $7.2 \mu \mathrm{m}$. The latter features are ascribed to aliphatic $\mathrm{CH}_{2} / \mathrm{CH}_{3}$ bending modes (e.g., Furton et al. 1999; Chiar et al. 2000; Dartois et al. 2007). Moreover, the strength of the $8.6 \mu \mathrm{m}$ feature, relative to the $\mathrm{CC}$ features at 6.2 and $7.7 \mu \mathrm{m}$, decreases with feature redshift.

Rapacioli et al. (2005), Berné et al. (2007), Joblin et al. (2008), and Berné et al. (2009) follow a different approach (see also Berné et al., elsewhere in this volume). This group has investigated the spatially resolved IR spectra of photodissociation regions (PDRs) and PNe, and found that they could be decomposed into a few fundamental basis spectra. Based on their fractional contribution to the emission in different spatial regions of PDRs, the fundamental spectra are attributed to different families of hydrocarbon molecules/grains: ionized PAHs, neutral PAHs and very small grains (VSGs; possibly PAH clusters: see Rapacioli et al., elsewhere in this volume). Berné et al. (2009) fit the observed PAH spectra of a sample of young stars with the basis spectra. They find that the fraction of the emission ascribed by the authors to VSGs decreases with increasing UV luminosity of the central star. This correlation is very likely the same as the one mentioned above between feature redshift and stellar effective temperature. The VSG spectrum used by Berné et al. contains the reddest features in their spectral basis. A reduction in the contributing fraction of the VSG spectrum is therefore equivalent to a blueshift of the features. The link between UV luminosity and $\mathrm{T}_{\text {eff }}$ is also clear: cooler stars have in general weaker photospheric UV fields. However, 
as opposed to the aliphatic/aromatic interpretation outlined above, Berné et al. suggest that an increased contribution of VSGs produces the position shift in the feature.

A third possible explanation, which has not been explored sufficiently in the literature, is that the $\mathrm{PAH}$ size and charge influence the spectral classification (Bauschlicher et al. 2008, 2009). The link between feature position and $\mathrm{T}_{\text {eff }}$ could then reflect the excitation and charge state of the PAHs with respect to the stellar radiation field.

Keller et al. (2008) find that the flux ratios of the 12.7 and $11.3 \mu \mathrm{m}$ features, and of the 7.7 and $11.3 \mu \mathrm{m}$ features correlate with stellar effective temperature. The 12.7/11.3 ratio probes the importance of aromatic duo/trio $\mathrm{CH}$ modes with respect to solo $\mathrm{CH}$ modes (Hony et al. 2001). The ratio is expected to be high in small PAHs, or PAHs with jagged edges. The features in the $6-9 \mu \mathrm{m}$ range are ascribed to ionized PAHs, and those at longer wavelengths to neutrals. The $7.7 / 11.3$ ratio is therefore interpreted as a measure for the ionization of the PAHs. Keller et al. (2008) conclude that PAH molecules around hotter stars have rougher edges and are more ionized, as a result of the stellar irradiation.

Finally, Visser et al. (2007) point out that the flux ratio of the 3.3 and $6.2 \mu \mathrm{m}$ features is another strong probe of the PAH charge. The ratio increases by an order of magnitude by going from ionized to neutral species. In the Herbig Ae/Be sample of Acke \& van den Ancker (2004), the detection rate of the $3.3 \mu \mathrm{m}$ feature, given the presence of the $6.2 \mu \mathrm{m}$ feature, is $44 \%$ : half of the PAH spectra are dominated by neutrals, the other half by ionized species. However, no link with stellar UV field or effective temperature is found.

\section{Conclusions}

The emitting hydrocarbon molecules in disks around young stars are different from those in the ISM, and their chemistry - aliphatic/aromatic hybridization ratio, clustering, size, and/or charge state - is connected to the stellar radiation field. There is an interplay between the disk structure and the strength of hydrocarbon emission. Targets with flared disks have brighter PAH features. In turn, the PAHs in the disk surface contribute to the gas thermal balance, shaping the disk morphology.

\section{References}

Acke, B., \& van den Ancker, M.E., 2004, A\&A, 426, 151

Acke, B., Min, M., van den Ancker, M.E., et al., 2009, A\&A, 502, L17

Acke, B., Bouwman, J., Juhász, A., et al., 2010, ApJ, 718, 558

Bauschlicher, C.W., Peeters, E., \& Allamandola, L.J., 2008, ApJ, 678, 316

Bauschlicher, C.W., Peeters, E., \& Allamandola, L.J., 2009, ApJ, 697, 311

Berné, O., Joblin, C., Deville, Y., et al., 2007, A\&A, 469, 575 
Berné, O., Joblin, C., Fuente, A., \& Ménard, F., 2009, A\&A, 495, 827

Boersma, C., Bouwman, J., Lahuis, F., et al., 2008, A\&A, 484, 241

Bouwman, J., Henning, T., Hillenbrand, L.A., et al., 2008, ApJ, 683, 479

Cesarsky, D., Lequeux, J., Ryter, C., \& Gérin, M., 2000, A\&A, 354, L87

Chiang, E.I., \& Goldreich, P., 1997, ApJ, 490, 368

Chiar, J.E., Tielens, A.G.G.M., Whittet, D.C.B., et al., 2000, ApJ, 537, 749

Dartois, E., Geballe, T.R., Pino, T., et al., 2007, A\&A, 463, 635

de Graauw, T., Haser, L.N., Beintema, D.A., et al., 1996, A\&A, 315, L49

Doucet, C., Habart, E., Pantin, E., et al., 2007, A\&A, 470, 625

Dullemond, C.P., Dominik, C., \& Natta, A., 2001, ApJ, 560, 957

Dullemond, C.P., \& Dominik, C., 2004, A\&A, 417, 159

Dullemond, C.P., Henning, T., Visser, R., et al., 2007, A\&A, 473, 457

Fedele, D., van den Ancker, M.E., Acke, B., et al., 2008, A\&A, 491, 809

Fedele, D., van den Ancker, M.E., Henning, T., et al., 2010, A\&A, 510, A72

Furlan, E., Hartmann, L., Calvet, N., et al., 2006, ApJS, 165, 568

Furton, D.G., Laiho, J.W., \& Witt, A.N., 1999, ApJ, 526, 752

Geers, V.C., Augereau, J., Pontoppidan, K.M., et al., 2006, A\&A, 459, 545

Geers, V.C., 2007, Ph.D. Thesis, Leiden Observatory, Leiden University

Geers, V.C., van Dishoeck, E.F., Visser, R., et al., 2007, A\&A, 476, 279

Geers, V.C., van Dishoeck, E.F., Pontoppidan, K.M., et al., 2009, A\&A, 495, 837

Habart, E., Verstraete, L., Boulanger, F., et al., 2001, A\&A, 373, 702

Habart, E., Natta, A., \& Krügel, E., 2004a, A\&A, 427, 179

Habart, E., Testi, L., Natta, A., \& Carbillet, M., 2004b, ApJ, 614, L129

Habart, E., Natta, A., Testi, L., \& Carbillet, M., 2006, A\&A, 449, 1067

Herbig, G.H., 1960, ApJS, 4, 337

Hony, S., Van Kerckhoven, C., Peeters, E., et al., 2001, A\&A, 370, 1030

Houck, J.R., Roellig, T.L., van Cleve, J., et al., 2004, ApJS, 154, 18

Joblin, C., Szczerba, R., Berné, O., \& Szyszka, C., 2008, A\&A, 490, 189

Keller, L.D., Sloan, G.C., Forrest, W.J., et al., 2008, ApJ, 684, 411

Kessler, M.F., Steinz, J.A., Anderegg, M.E., et al., 1996, A\&A, 315, L27

Lagage, P.O., Doucet, C., Pantin, E., et al., 2004, The Messenger, 117, 12

Lagage, P., Pel, J.W., Authier, M., et al., 2006, Science, 314, 621

Meeus, G., Waters, L.B.F.M., Bouwman, J., et al., 2001, A\&A, 365, 476

Meeus, G., Pinte, C., Woitke, P., et al., 2010, A\&A, 518, 124

Meijer, J., Dominik, C., de Koter, A., et al., 2008, A\&A, 492, 451

Oliveira, I., Pontoppidan, K.M., Merín, B., et al., 2010, ApJ, 714, 778

Peeters, E., Hony, S., Van Kerckhoven, C., et al., 2002, A\&A, 390, 1089

Pino, T., Dartois, E., Cao, A., et al., 2008, A\&A, 490, 665

Preibisch, T., Kim, Y., Favata, F., et al., 2005, ApJS, 160, 401

Quanz, S.P., Henning, T., Bouwman, J., et al., 2007, ApJ, 668, 359

Rapacioli, M., Joblin, C., \& Boissel, P., 2005, A\&A, 429, 193

Siebenmorgen, R., \& Krügel, E., 2010, A\&A, 511, A6 
Sloan, G.C., Keller, L.D., Forrest, W.J., et al., 2005, ApJ, 632, 956

Sloan, G.C., Jura, M., Duley, W.W., et al., 2007, ApJ, 664, 1144

Sloan, G.C., 2008, in IAU Symposium, ed. S. Kwok \& S. Sandford, 251, 191

Stelzer, B., Micela, G., Hamaguchi, K., \& Schmitt, J.H.M.M., 2006, A\&A, 457, 223

van Boekel, R., Waters, L.B.F.M., Dominik, C., et al., 2004, A\&A, 418, 177

van der Plas, G., van den Ancker, M.E., Fedele, D., et al., 2008, A\&A, 485, 487

Verhoeff, A.P., Min, M., Acke, B., et al., 2010, A\&A, 516, A48

Visser, R., Geers, V.C., Dullemond, C.P., et al., 2007, A\&A, 466, 229 